\title{
Shared Savings Models for ACOs-Incentivizing Primary Care Physicians
}

\author{
Mona Siddiqui, MD, MPH and Scott A. Berkowitz, MD, MBA $A^{1,2}$ \\ 'Department of Medicine, Johns Hopkins University School of Medicine, Baltimore, MD, USA; ${ }^{2}$ Division of Cardiology, Johns Hopkins University \\ School of Medicine, Baltimore, MD, USA.
}

J Gen Intern Med 29(6):832-4

DOI: $10.1007 / \mathrm{s} 11606-013-2733-5$

(c) Society of General Internal Medicine 2013

I ncreasing numbers of health systems and physician practices are considering formation of accountable care organizations (ACOs) to promote clinical integration and to create a potential revenue stream through shared savings. In large part, the ACO triple aim of improved experience of care, better population health and decreased cost is to be achieved by empowering primary care physicians who will be the "quarterbacks" for preventive care and care coordination and will serve as the main source of attribution for patients to an ACO. As competing ACOs are established within overlapping markets, an ACO's success will be determined in large part by its ability to attract and retain a vibrant and effective primary care workforce, currently experiencing a national shortage. Although the potential for financial incentives through shared savings is one way in which ACOs can attract primary care providers, there is currently no agreed upon taxonomy for categorizing or evaluating shared savings distribution methodologies and their impact on primary care providers and organizational success. Herein we propose a taxonomy of shared savings decision-making and discuss four models for shared savings distribution that recognize the increasing importance of primary care within ACOs.

The Medicare Shared Savings Program (MSSP) defines the process for calculating shared savings at the level of the ACO, but provides little guidance regarding "optimal" allocation of savings at the provider level to incentivize physicians. Per the MSSP final rule, the Centers for Medicare and Medicaid Services (CMS) believes this flexibility will enable "innovation and achievement of an incentive payment while focusing on the three-part aim."1 Inherent in this assertion is that financial incentives should be tied to metrics of performance at the provider level. As provider level performance tracking becomes increasingly refined, metrics of quality will certainly impact allocation of shared savings. Primary care providers must, therefore, understand quality metrics that will be used to evaluate their

Published online December 24, 2013 performance as well as the ways in which these metrics and eventually measures of cost will be tied to their compensation.

Organizations able to generate savings will have at least three distinct allocation decisions, which we define as three "tiers". The first tier decision addresses savings allocation for operating budgets and infrastructure reinvestment versus provider incentives. The second tier decision focuses on division of incentives among provider groups, including, but not limited to: primary care physicians, specialists, and hospitals or physicians providing care for ACO patients, but unlikely to have attributed patients by the Medicare ACO formula. To address this second tier, some ACOs will determine fixed ratios among provider groups or focus on smaller subsets. Finally, the third tier decision reflects allocation of incentives within provider groups. We describe several different models of approaching this third tier allocation based on three overarching goals.

First, we propose that any approach should promote cross-specialty collaboration and "de-silo" departments that have historically thrived in their independence. ${ }^{2}$ Second, incentives should be sensitive to individual-level behavior. Third, to promote culture change, any methodology needs to have buy-in from relevant stakeholders, including primary care and specialty care physicians. Although related models exist commercially, they have yet to be promoted as an approach within Medicare. Moreover, most of these incentive design strategies are deemed proprietary, the result of negotiation, and little is publicly available regarding their implementation or outcomes. ${ }^{3}$ Currently, there is no consistent distribution strategy employed by ACOs. Given the historic mixed results of pay for performance strategies, we believe that defining a taxonomy is an important first step in weighing the potential benefits and drawbacks as well as in establishing a common framework for evaluation of the impact on provider practice and patient outcomes. ${ }^{4}$ We propose four models that ACOs may consider (Table 1): (1) an Attribution Incentive Model, (2) an Incremental Incentive Model, (3) a Threshold Incentive Model, and (4) an Endowment Incentive Model.

In an Attribution Incentive Model, ACO shared savings may be distributed among all physicians with attributed patients. If the health system attains savings, the proportion allocated for physicians may be distributed based on 
Table 1. Key Features of ACO Incentive Models for Primary Care Physicians

\begin{tabular}{|c|c|c|c|c|}
\hline & $\begin{array}{l}\text { Attribution Incentive } \\
\text { Model }\end{array}$ & $\begin{array}{l}\text { Incremental Incentive } \\
\text { Model }\end{array}$ & $\begin{array}{l}\text { Threshold Incentive } \\
\text { Model }\end{array}$ & $\begin{array}{l}\text { Endowment Incentive } \\
\text { Model }\end{array}$ \\
\hline Key Model Characteristics & $\begin{array}{l}\text { Incentives based only } \\
\text { on number of } \\
\text { beneficiaries per } \\
\text { primary care provider }\end{array}$ & $\begin{array}{l}\text { Incentives based } \\
\text { on incremental } \\
\text { improvement } \\
\text { in quality }\end{array}$ & $\begin{array}{l}\text { Incentives determined by } \\
\text { meeting both quality } \\
\text { and cost thresholds } \\
\text { or "gates" }\end{array}$ & $\begin{array}{l}\text { Upfront incentives with } \\
\text { threat of "take back" } \\
\text { if quality and cost } \\
\text { benchmarks are not met }\end{array}$ \\
\hline $\begin{array}{l}\text { Promotes individual } \\
\text { behavior change }\end{array}$ & & + & ++ & +++ \\
\hline $\begin{array}{l}\text { Promotes cross-specialty } \\
\text { collaboration }\end{array}$ & - & + & ++ & +++ \\
\hline Analytic requirements & + & ++ & +++ & +++ \\
\hline Overall considerations & $\begin{array}{l}\text { Most simple to implement; } \\
\text { incentivizes volume } \\
\text { but not linked to quality } \\
\text { or cost }\end{array}$ & $\begin{array}{l}\text { Links incentives to } \\
\text { quality; Requirement } \\
\text { for greater analytic } \\
\text { capability than random } \\
\text { sampling requested } \\
\text { by CMS }\end{array}$ & $\begin{array}{l}\text { Links incentives to both } \\
\text { quality and cost; could } \\
\text { be considered as initial } \\
\text { model to implement } \\
\text { if analytic requirements } \\
\text { are met }\end{array}$ & $\begin{array}{l}\text { Links incentives to both } \\
\text { quality and cost; would } \\
\text { require strong provider } \\
\text { buy-in; could be overlaid } \\
\text { on to the other models }\end{array}$ \\
\hline
\end{tabular}

number of beneficiaries attributed to the provider regardless of individual level performance. This formula would be simple to administer, with or without complex analytic capability, and may promote primary care productivity (incentivized to provide excellent primary care to more Medicare beneficiaries to increase the number attributed). Rewarding providers based solely on attribution volume, however, would exclude many specialty care or "non-attributed" physicians and may have little impact on cross specialty coordination. Incentives not aligned with performance could also lack responsiveness to individual-level behavior needed to motivate low performers to improve.

An Incremental Quality Incentive Model would grade provider performance on the 33 quality measures specified by CMS as well as any others deemed by the ACO to be of high institutional priority. Providers with attributed patients demonstrating even marginal quality improvement would be eligible for savings, incrementally, to ensure incentives are commensurate with performance gains, although, as above, incentives would be weighted towards providers with larger attributed patient pools. This strategy would superimpose some value for individual high-quality care and improvement. There are two potential downsides. First, assessing incremental improvements for measures such as those in the Group Practice Reporting Option may require more comprehensive data and analytic capability than the sampling requested by CMS, and institutional health information technology capabilities would be important to ensure changes are not due to random variation. Second, a system based on rewarding improvements rather than targeting defined outcomes may reward low performers making minimal gains at the expense of initial high performers with little room for marginal gains and thus may not foster cooperation or a sense of fairness.

A Threshold Incentive Model based on measures of quality and average per patient cost with eligibility thresholds or "gates" for savings may be an alternative to a strategy that rewards incremental improvement. Prespecified quality and cost thresholds, or a scorecard of such measures, would create a system that would be fair and transparent to administer and may lead to more rapid and sustained performance gains depending on the level of the "gates". Attribution weighting could be used as before, but there is also the potential to apply this methodology for non-attributed providers, such as hospitalists, radiologists, emergency department physicians, and others. The "gates" could be modified yearly to ensure that thresholds remain relevant to institutional goals. This strategy would be responsive to individual practice patterns and would promote cross specialty collaboration, although a cost "gate" may not be a transparent benchmark and the complexity of implementing this model may be a drawback. An additional limitation may be its impact on low performers who may not realize any savings for performance improvements until threshold is reached, while high performers will be eligible without demonstrating improvements. While incentivizing based on cost of care may cause concerns regarding patient selection and increasing disparities, such a practice is expressly forbidden and will be carefully monitored by CMS. Appropriate risk adjustment and comparisons with previous years would further help mitigate the risk. Careful selection of measures of cost will be critical to validate that savings truly reflect evidencegenerated improvements in care.

Finally, an Endowment Incentive Model, based on the economic principle that individuals go to greater lengths to avoid losses than to obtain gains of an equivalent magnitude, would provide up-front incentive support to providers, based on historical data, with the threat of takeback if defined quality and cost measures are not ultimately met. ${ }^{5}$ While a drawback may be the requirement for initial capital investment, either from institutional investment or capitation payments (not available with the MSSP at this time), an aversion to losing this "up-front" incentive would likely promote individual behavior change and cross- 
specialty collaboration. Institutional analytic capability would be necessary to determine up-front allocation and to reconcile payments at performance year end. As ACOs gain experience with implementing incentives, the Endowment Incentive Model could be overlaid with preceding strategies.

The first three incentive models represent a gradation in increasing analytic complexity for implementation, while the fourth requires significant advanced planning and investment. Since providers may respond best to incentive strategies that reflect individual performance, we favor pursuing the most advanced analytic model that could be effectively implemented. Therefore, when possible, and until further evaluation allows for better comparison between the models and associated efficacy, we can see value in pursuing the Threshold Incentive Model. For the Endowment Incentive Model, we favor first gaining initial program experience.

Three-tier decision-making and a taxonomy for shared savings can create a framework for approaching CMS ACO arrangements. Each ACO will have unique needs and capabilities that will impact the approach selected, and a common language is necessary to properly evaluate the impact of these incentive strategies. To motivate system culture change and improve outcomes, ACO incentives should reward primary care physicians who are stewards of preventive care and care coordination. Educating primary care providers on opportunities with respect to shared savings will empower them to make appropriate choices while facilitating collaboration to achieve optimal patient outcomes.

Conflict of Interest: The authors have no financial disclosures or conflicts of interest.

Corresponding Author: Scott A. Berkowitz, MD, MBA; Division of Cardiology, Johns Hopkins University School of Medicine, $600 \mathrm{~N}$. Wolfe Street, Billings Administration-Room 327A, Baltimore, MD 21287, USA (e-mail: sberkow3@jhmi.edu).

\section{REFERENCES}

1. Department of Health and Human Services, Centers for Medicare \& Medicaid Services. Medicare program; Medicare Shared Savings Program: Accountable Care Organizations. Final rule. Fed Regist. 2011;76(212):6780267990.

2. Berkowitz SA, Miller ED. Accountable care at academic medical centers-lessons from Johns Hopkins. New England Journal of Medicine. 2011;364(7).

3. Weissman JS, Bailit M, D'Andrea G, Rosenthal MB. The design and application of shared savings programs: lessons from early adopters. Health Affairs. 2012;31(9):1959-1968.

4. Ryan AM, Blustein J, Casalino LP. Medicare's flagship test of pay-forperformance did not spur more rapid quality improvement among lowperforming hospitals. Health Aff. 2012;31(4):797-805.

5. Kahneman D, Knetsch J, Thaler R. Experimental tests of the endowment effect and the coase theorem. Journal of Political Economy. 1990: 1325-1348 